\title{
Peptide-Functionalized Nanostructured Microarchitectures Enable Rapid Mechanotransductive Differentiation
}

\author{
Zongjie Wang ${ }^{1,2, \#, ~ L i b i n g ~ Z h a n g ~}{ }^{3, \#}$, Mahmoud Labib ${ }^{3}$, Haijie Chen ${ }^{1}$, Mingyang Wei ${ }^{1}$, Mahla \\ Poudineh ${ }^{3}$, Brenda J. Green ${ }^{2}$, Bill Duong ${ }^{4}$, Jagotamoy Das ${ }^{3}$, Sharif Ahmed ${ }^{3}$, Edward $H$. \\ Sargent ${ }^{1}$ and Shana O. Kelley ${ }^{2,3,4^{*}}$
}

${ }^{1}$ The Edward S. Rogers Sr. Department of Electrical \& Computer Engineering, University of Toronto, Toronto, M5S 3G4, Canada. ${ }^{2}$ Institute for Biomaterials and Biomedical Engineering, University of Toronto, Toronto, M5S 3G9, Canada. ${ }^{3}$ Department of Pharmaceutical Sciences, Leslie Dan Faculty of Pharmacy, University of Toronto, Toronto, M5S 3M2, Canada. ${ }^{4}$ Department of Biochemistry, Faculty of Medicine, University of Toronto, Toronto, M5S 1A8, Canada. \#: These authors contribute equally to this work. Correspondence to: shana.kelley@utoronto.ca 


\section{Materials and Methods}

Chip Fabrication. Glass substrates coated with $20 \mathrm{~nm} \mathrm{Cr}, 150 \mathrm{~nm}$ Au were purchased from EMF Corp. (Ithaca, NY). The glass substrates were spin-coated with S1813 positive photoresist (MicroChem, Westborough, MA) at $2000 \mathrm{rpm}$ for 30s. The coated substrates were exposed to an ultraviolet (UV) light source (MA6 mask aligner, Karl Suss, Garching, Germany) for 10s and developed in MF321 (Dow Corning, Midland, MI) to selectively pattern the photoresist. Gold electrodes were obtained via etching away the $\mathrm{Cr}$ and $\mathrm{Au}$ areas that were not covered by S1813. Residues of S1813 were completed removed by an AZ300T stripper (AZ Electronics Materials, Somerville, NJ). The gold electrodes were then spin-coated with SU8-2002 negative photoresist (MicroChem) at $3000 \mathrm{rpm}$ for 40s. SU-8 layer was exposed to the UV light source for 12s and developed in SU8 developer (MicroChem) for 20 s to have an array of $5 \mu \mathrm{m}$ wide linear apertures on the gold electrodes. Patterned substrates were then diced by DISCO dicing saw (Tokyo, Japan) to make multiple chips.

Electrodeposition. Nanostructured architectures were electrodeposited to the chips via direct current potential amperometry (DCPA) via a two-step process. First, gold microarchitectures were deposited at $0 \mathrm{~V}$ for $100 \mathrm{~s}$ in a solution of $50 \mathrm{mM}$ $\mathrm{HAuCl}_{4}$ (99.99\% trace metals basis, Sigma-Aldrich, St. Louis, MO) and $0.5 \mathrm{M} \mathrm{HCl}$. Subsequently, gold nanoarchitectures were deposited at $-0.4 \mathrm{~V}$ for $30 \mathrm{~s}$ in a solution of $20 \mathrm{mM} \mathrm{HAuCl}_{4}$ and $0.5 \mathrm{M} \mathrm{HClO}_{4}$.

RGD functionalization. Chips were rinsed with acetone, isopropyl alcohol and DI water and dried by nitrogen before conjugation. Self-assembled monolayers were prepared by immersing the chips in $1.0 \mathrm{mM}$ ethanolic solutions of MUA for $12 \mathrm{~h}$. The chips then were washed with ethanol and ultrapure water and immersed in a solution of $5 \mathrm{mg} / \mathrm{mL} \quad \mathrm{N}$-(3-Dimethylaminopropyl)-N'-ethylcarbodiimide hydrochloride (98.0\%, Sigma-Aldrich) and $5 \mathrm{mg} / \mathrm{mL} \mathrm{N}$-hydroxysuccinimide (98\%, Sigma-Aldrich) in ultrapure water $(\mathrm{pH}=7)$ for 1.0 hour. The solution was stirred every 15 mins to remove the bubbles. Subsequently, the chips were rinsed with ultrapure water and immersed in an aqueous solution of $1.0 \mathrm{mM}$ Gly-Arg-Gly-Asp- 
Ser (RGD, >97\%, ab142698, Abcam, Cambridge, UK) overnight. The modified chips were rinsed three time with ultrapure water before use. Geltrex was purchased from Thermo Fisher Scientific (A1569601, Waltham, MA) in a ready-touse format. $0.5 \mathrm{~mL}$ of Geltrex solution was added onto the chips and incubated at $37^{\circ} \mathrm{C}$ for 2 hours before use.

Atomic force microscopy (AFM). The thickness of functionalized gold surface was measured by an Asylum Research Cypher AFM equipped with AC240TM-R3 probes (resonant frequency $70 \mathrm{kHz}$, spring constant $2 \mathrm{~N} \mathrm{~m}^{-1}$ ). Nanomechanical force spectroscopy was collected in which a trigger indentation force of $8 \mathrm{nN}$ was loaded at a rate of $2 \mu \mathrm{m} \mathrm{s}^{-1}$. The thickness was calculated by comparing the spectra of the bare gold and RGD-functionalized gold surfaces.

Density of functionalization. The density of functionalization on the gold surface was calculated according to the reductive desorption method. The reductive desorption Au-S bond was obtained using linear sweep voltammetry in $0.05 \mathrm{M}$ $\mathrm{KOH}$ solution (Fig. S2).

The reaction shown below:

$$
\mathrm{Au}-\mathrm{SR}+\mathrm{e}^{-} \rightarrow \mathrm{Au}+\mathrm{SR}^{-}
$$

Integrating the reduction peak (background subtracted), the surface density of MUA (Г) was calculated according to the previously established relationship as displayed in the following Equation (2).

$$
\Gamma=Q / n F A
$$

Where, $Q$ is the charge required to reduce the Au-S bond, $n$ is the number of electrons per molecule reduction, $F$ is the Faraday constant (C/equiv), and $A$ is the electrode area $\left(\mathrm{cm}^{2}\right)$.

Culture chamber assembly. Polydimethylsiloxane (PDMS, Sylgard 184, Dow Corning, Midland, MI) was mixed at 10:1 ratio with its curing agent (Dow Corning), placed on a 3D printed mold (printed by UPrint, Stratasys, Eden Prairie, MN) and cured at $70^{\circ} \mathrm{C}$ for overnight to generate chambers for cell culturing. Chambers and chips were clamped by laser-cut acrylic boards and bonded mechanically via 
screwing. This bonding was reversible via loosing screws to allow downstream imaging process.

Cell culture. Bone marrow-derived human mesenchymal stem cells (HMSCs) were purchased from Cyagen (Santa Clara, CA) and cultured in HMSC growth medium (Cyagen). The medium was changed twice a week. The passage number of HMSC used in the experiments was lower than 15. During experiments, cells were trypsinized, centrifuged and redistributed to the chambers for further experiments. The cell concentration for each experiment was listed in Table S1.

Scanning electron microscopy (SEM). HMSCs were fixed by $2 \%$ glutaraldehyde in $0.1 \mathrm{M}$ sodium cacodylate buffer for 2 hours and rinsed with $0.1 \mathrm{M}$ sodium cacodylate buffer with $0.2 \mathrm{M}$ sucrose for 20 mins. Fixed samples were dehydrated by ethanol and the critical point drying procedure was performed (CPD 030, Leica, Wetzlar, Germany). Chips with and without cells were sputter coated with $15 \mathrm{~nm}$ Au (Denton Desk II, Leica) and imaged under a SU5000 field emission SEM (Hitachi, Tokyo, Japan) using 5kV accelerating voltage. Pseudo-colored SEM images were obtained by manually processing images in Photoshop (Adobe, San Jose, CA).

Immunostaining. To access the cell viability on chips, HMSCs were stained by 4 drops Hoechst 33342 (H33342, Invitrogen), $1 \mu \mathrm{M}$ calcein acetoxymethyl (Biotium, Fremont, CA) and $4 \mu \mathrm{M}$ ethidium homodimer-III (EthD-III, Biotium) in $1 \mathrm{~mL} 1 \%$ BSA in phosphate-buffered saline (PBS) for 30 mins. Cell viability was quantified by fluorescence microscopy. To access the viability of released cells, HMSCs in buffer were pipetted well and mixed with $0.4 \%$ Trypan blue solution (Thermo Fisher). Cell number and viability were quantified by an automated cell counter (Countess II, Thermo Fisher).

To access the protein expression on chips or well plates, HMSCs were fixed by $4 \%$ methanol-free paraformaldehyde for 10 mins, permeabilized by $0.2 \%$ Triton X100 for 10 mins, blocked by $1 \%$ bovine serum albumin in PBS and then stained by the combination of antibodies in 1\% BSA, $0.1 \%$ Tween 20 in PBS. Detailed information of antibodies was given in Table S2. 
Fluorescence microscopy and image processing. Stained samples were observed under an inverted microscope (Ti-E, Nikon, Tokyo, Japan) with 10X, 20X and 50X ELWD lens.

The nucleus counting function in WCIF ImageJ (University Health Network, Toronto, ON) was utilized to quantify total cell number (through H33342) and dead cell number (EthD-III). On-chip cell viability was calculated based on the ratio of total cell number and dead cell number. The body length of the individual released cells was also measured via WCIF ImageJ.

MATLAB 2017b (Mathworks, Natick, MA) was utilized to quantify the percentage of MAP2 positive cells. Thresholds were manually set to binarize the DAPI and MAP2 images. The binarized images were colocalized to find $\mathrm{DAPI}(+)$ and MAP2(+) areas. Particle counting functions were used to determine the total number of cells $(\mathrm{DAPI}(+))$ and MAP2 positive cells (DAPI(+)-MAP2(+)). Areas with a size smaller than $80 \mu \mathrm{m} 2$ were not considered as valid cells.

\section{Reverse transcription-quantitative polymerase chain reaction (RT-qPCR)} Total RNA was isolated from the cells by using single-cell RNA purification kit (51800, Norgen Biotek, Thorold, ON, Canada) and used for RT-qPCR. The isolated RNA was used for cDNA synthesis using the first strand DNA synthesis kit (Invitrogen), which contained random hexamer primers and Superscript III reverse transcriptase, according to the manufacturer's protocol. A comparative $\mathrm{Ct}$ experiment was performed on ViiA ${ }^{\mathrm{TM}} 7$ real-time PCR (Life Technologies, US). Detailed information of antibodies was given in Table S3. GAPDH was utilized as a housekeeping gene control. All nucleic acid sequences were purchased from Integrated DNA Technologies (Coralville, IA). The assay was carried out in triplicates using $10 \mathrm{ng}$ cDNA for each sample in a 96-well plate. The $20 \mu \mathrm{L}$ reaction mix consisted of $10 \mu \mathrm{L}$ of SsoFast EvaGreen master mix (Bio-Rad, Hercules, CA), $0.5 \mu \mathrm{L}$ of forward primer, $0.5 \mu \mathrm{L}$ of reverse primer, $8 \mu \mathrm{L}$ of water and $1 \mu \mathrm{L}$ of $10 \mathrm{ng}$ $\mu \mathrm{L}-1 \mathrm{cDNA}$. Cycling conditions for the qPCR were $95^{\circ} \mathrm{C}$ for $10 \mathrm{~min}$, followed by 40 cycles of $95^{\circ} \mathrm{C}$ for $15 \mathrm{~s}$ and $60^{\circ} \mathrm{C}$ for $1 \mathrm{~min}$. 
Flow cytometry. HMSCs were recovered by $0.25 \%$ trypsin for 3 mins, fixed by $4 \%$ methanol-free paraformaldehyde for 10 mins, permeabilized by $0.5 \%$ Triton X-100 for 10 mins, blocked by $1 \%$ bovine serum albumin in PBS and then stained by the combination of antibodies in 1\% BSA, $0.1 \%$ Tween 20 in PBS. Detailed information of antibodies used in flow cytometry was given in Table S4. Stained samples, together with unstained samples and isotype controls were examined immediately after staining by a flow cytometer (FACS Canto, BD Biosciences, Franklin Lakes, $\mathrm{NJ}$ ). Acquired data was analyzed by FlowJo software (FlowJo LLC., Ashland, OR).

Statistical analysis. A one-way analysis of variance (ANOVA analysis) function in MATLAB 2017b was used to statistically analyze the quantitative data. Results were shown as an average \pm standard deviation.

\section{Tables}

Table S1. Cell conditions in different experiments

\begin{tabular}{|c|c|}
\hline Experiment/Markers & Cell number per device \\
\hline On-chip cell viability & 25,000 \\
\hline On-chip proliferation (Ki67) & 25,000 \\
\hline On-chip Tuj1/MAP2 & 25,000 \\
\hline On-chip Integrin/pFAK & 25,000 \\
\hline Cell recovery for western blotting & 50,000 \\
\hline Cell recovery characterization & 25,000 \\
\hline Cell recovery for culture & 25,000 \\
\hline Cell recovery for flow cytometry & 50,000 \\
\hline
\end{tabular}

Table S2. Details of antibodies for immunostaining

\begin{tabular}{|l|l|l|l|l|l|l|}
\hline Antibody & Host & Conjugation & Dilution & Duration & Supplier & Cat. \# \\
\hline DAPI & N/A & N/A & 4 drops/mL & 30 mins & Invitrogen & R37606 \\
\hline F-actin & N/A & AF 555 & $7: 500$ & 30 mins & Cytoskeleton & PHDH1 \\
\hline Ki67 & Rabbit & AF 647 & $1: 100$ & 2 hours & Abcam & ab196907 \\
\hline Tuj-1 & Rabbit & AF 488 & $1: 100$ & 2 hours & Abcam & Ab185031 \\
\hline
\end{tabular}




\begin{tabular}{|l|l|l|l|l|l|l|}
\hline MAP2 & Rabbit & APC & $1: 100$ & 2 hours & LSBio & C223150 \\
\hline Integrin & Mouse & Unconjugated & $1: 100$ & 2 hours & Abcam & ab24693 \\
\hline pFAK & Rabbit & Unconjugated & $1: 100$ & 2 hours & Thermo & 700255 \\
\hline Anti rabbit & Donkey & AF 488 & $1: 200$ & 1 hour & Abcam & ab150073 \\
\hline Anti mouse & Donkey & AF 647 & $1: 200$ & 1 hour & Abcam & ab150107 \\
\hline
\end{tabular}

AF: Alexa Fluor, APC: allophycocyanin

Table S3. Details of primers used for qPCR

\begin{tabular}{|l|l|l|}
\hline Gene & Forward primer & Reverse primer \\
\hline MAP2 & CTTCAGCTTGTCTCTAACCGAG & CCTTTGCTTCATCTTTCCGTTCG \\
\hline Ki67 & ACGCCTGGTTACTATCAAAAGG & CAGACCCATTTACTTGTGTTGGA \\
\hline NeuN & CCAAGCGGCTACACGTCTC & CGTCCCATTCAGCTTCTCCC \\
\hline GAPDH & CATGAGAAGTATGACAACAGCCT & AGTCCTTCCACGATACCAAAGT \\
\hline
\end{tabular}

Table S4. Details of antibodies used for flow cytometry

\begin{tabular}{|l|l|l|l|l|l|l|}
\hline Antibody & Host & Conjugation & Dilution & Duration & Supplier & Cat. \# \\
\hline Islet1 & Mouse & Unconjugated & $1: 200$ & 2 hours & Novus & NBP1-47537 \\
\hline GABA & Rabbit & Unconjugated & $1: 200$ & 2 hours & Novus & NB300-199 \\
\hline TH & Mouse & Unconjugated & $1: 200$ & 2 hours & Novus & MAB7566 \\
\hline TPH-2 & Rabbit & Unconjugated & $1: 200$ & 2 hours & Novus & NB100-74555 \\
\hline Anti rabbit & Donkey & AF 488 & $1: 500$ & 1 hour & Abcam & ab150073 \\
\hline Anti mouse & Donkey & AF 647 & $1: 500$ & 1 hour & Abcam & ab150107 \\
\hline
\end{tabular}

AF: Alexa Fluor 


\section{Figures}
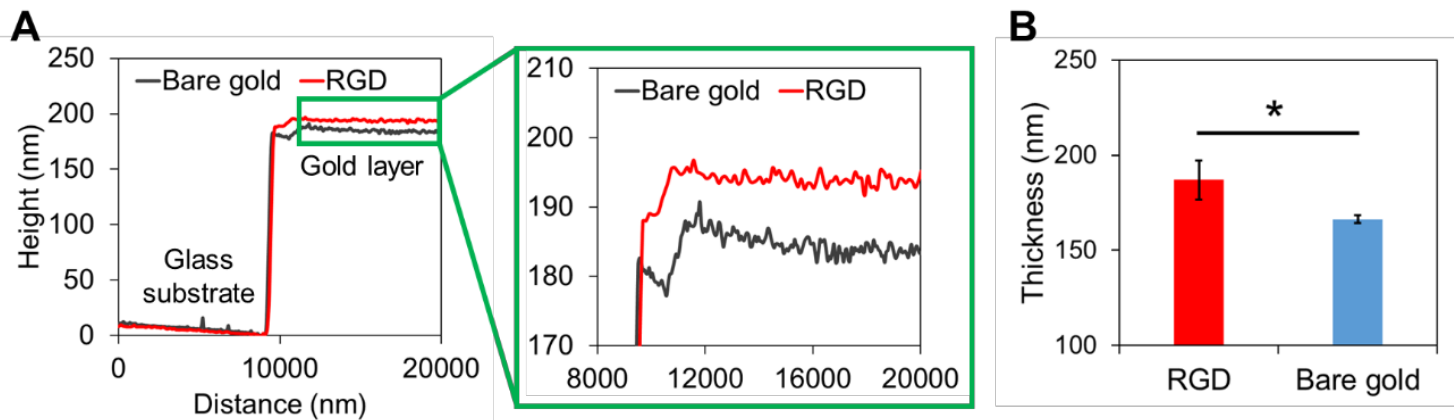

Figure S1. Determination of the thickness of the functionalized gold surface. (A) Representative curves of thickness for bare gold and RGD-functionalized surfaces acquired by AFM. (B) Quantification of thickness. RGD functionalized gold surfaces were significantly thicker than the bare gold surfaces $\left(n=3,{ }^{*} p<0.05\right)$.

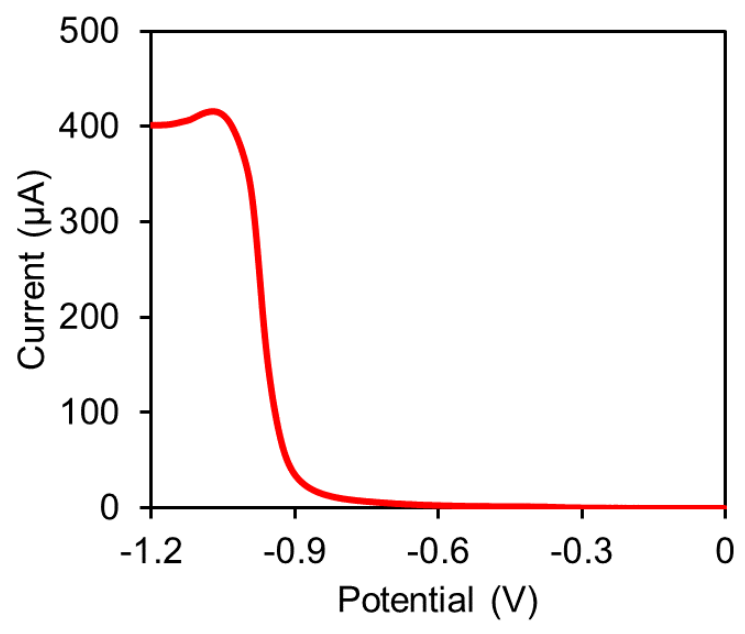

Figure S2. Quantification of the density of functionalization via measuring the reductive desorption of MUA in $0.05 \mathrm{M} \mathrm{KOH}, 0.02 \mathrm{~V} / \mathrm{s}$ vs. Ag/AgCl. 


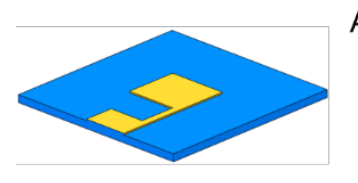

Attach PDMS
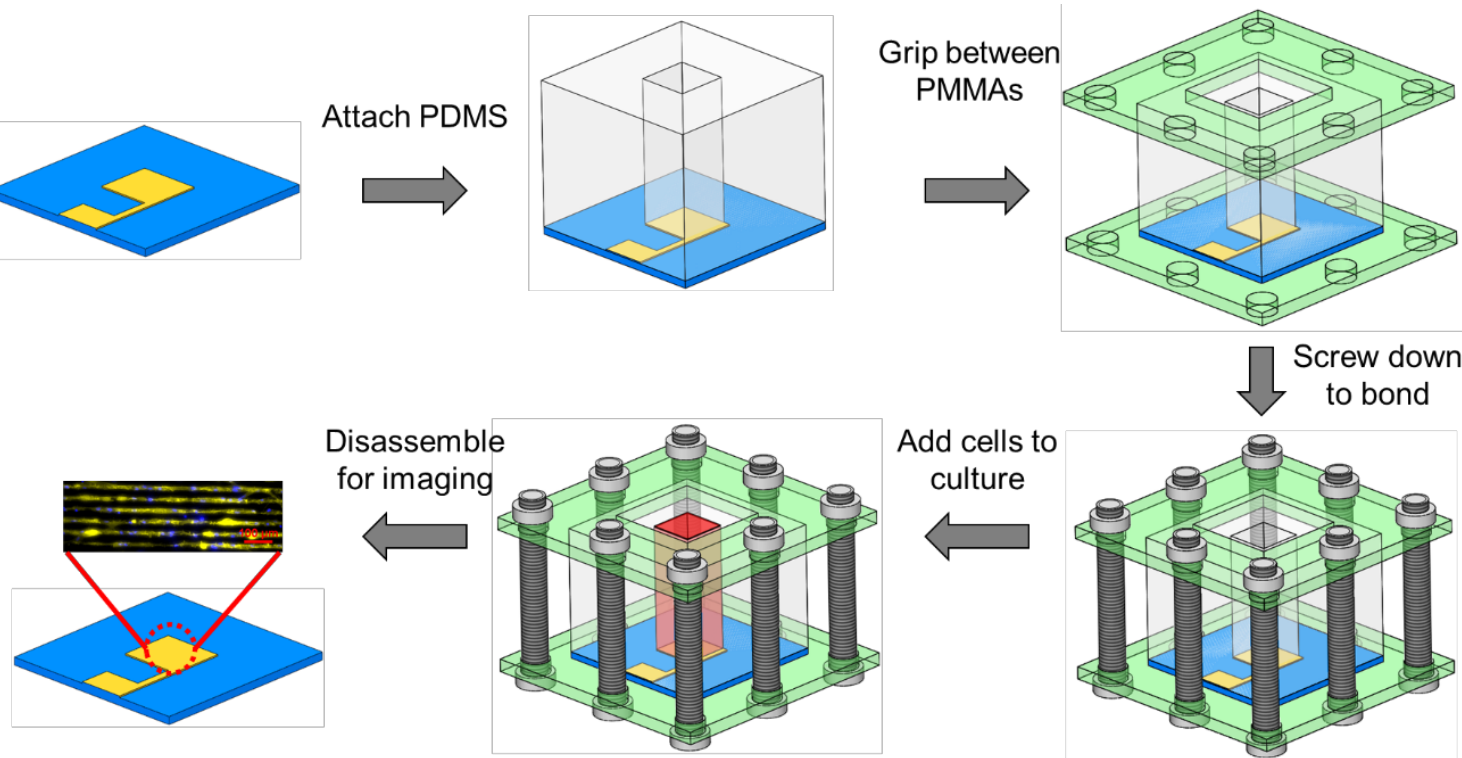

Figure S3. Reversible bonded devices for culturing and imaging of hMSCs on nanostructured architectures. PDMS chamber was attached to the slide and sealed mechanically via tightening PMMA boards. After 7 days of culture, the device was disassembled for immunostaining or SEM imaging.
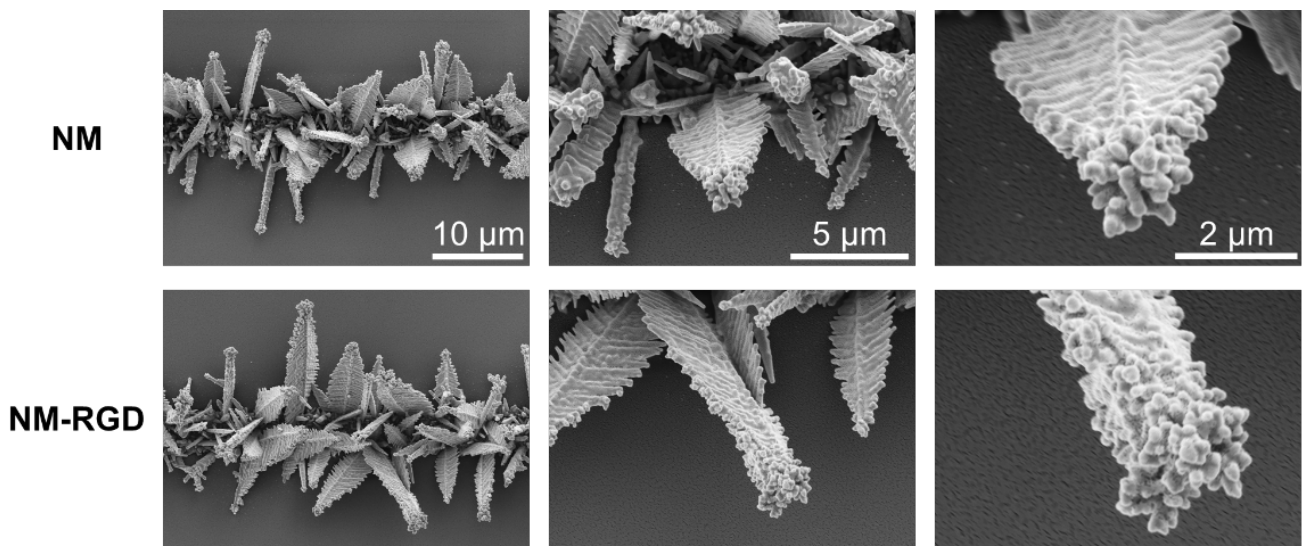

Figure S4. SEM images of the nanostructured architectures wi/wo RGD conjugation. Both architectures contain 3D leaf structures with a large population of nano-needles. The conjugation process did not affect the sharpness of nano-needles. 


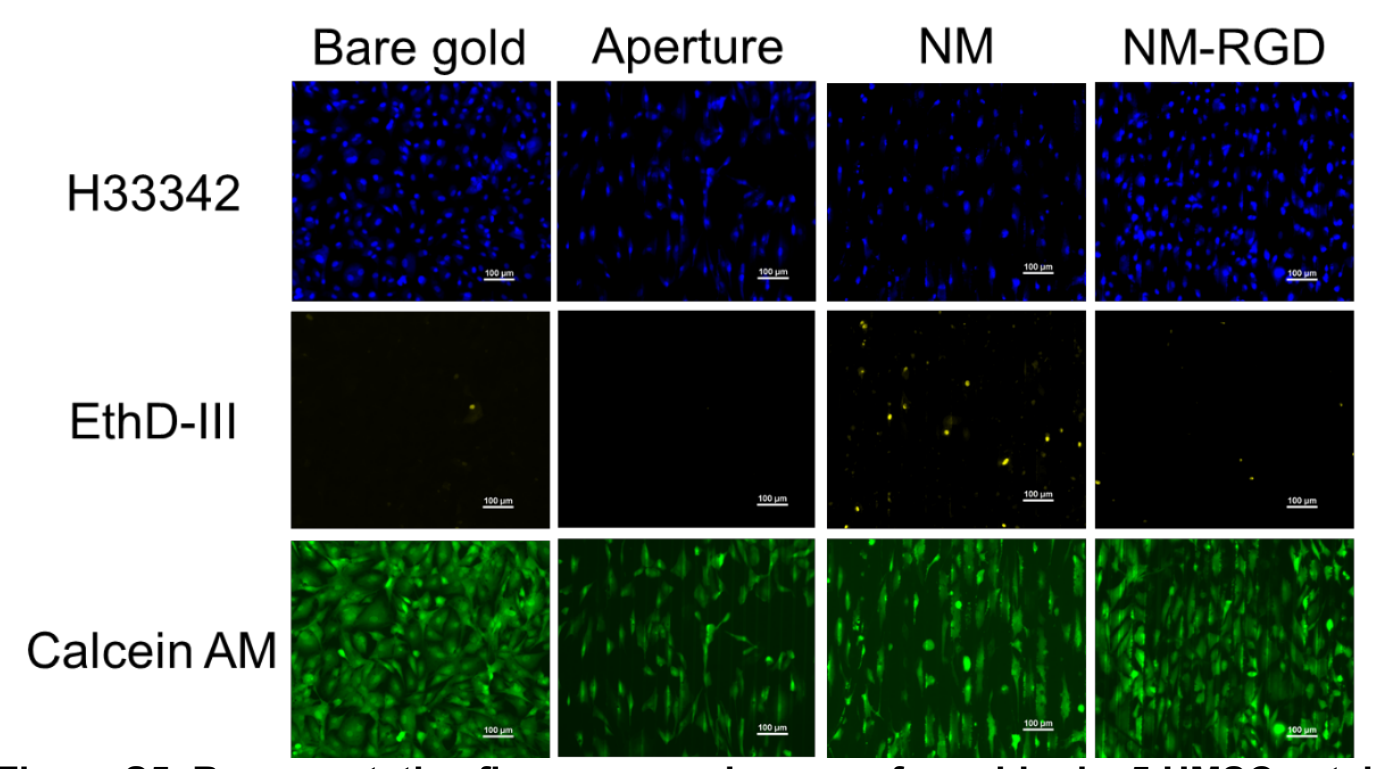

Figure S5. Representative fluorescence images of on-chip day5 HMSCs stained by live/dead assay. More dead cells (EthD-III(+)) were found on NM substrate.

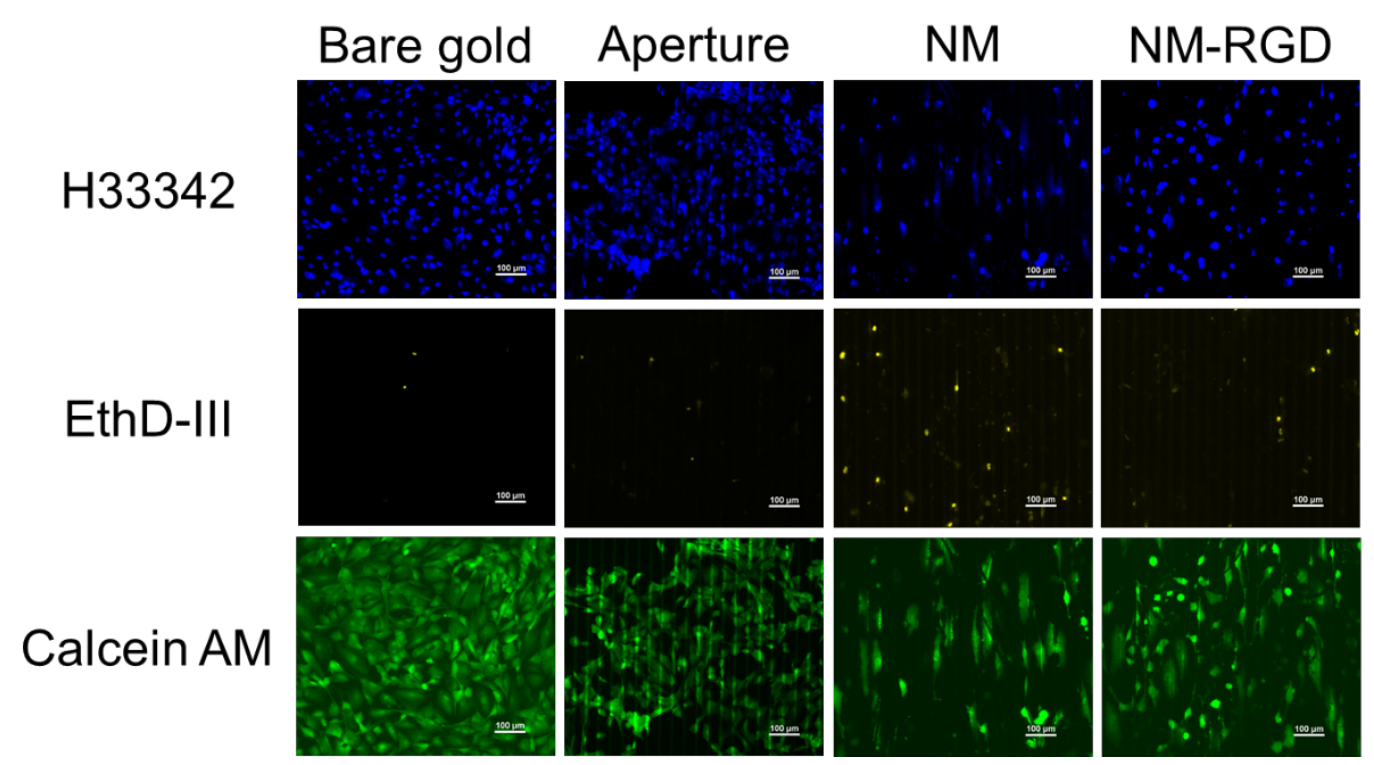

Figure S6. Representative fluorescence images of on-chip day10 HMSCs stained by live/dead assay. More dead cells (EthD-III(+)) were found on NM substrate. 

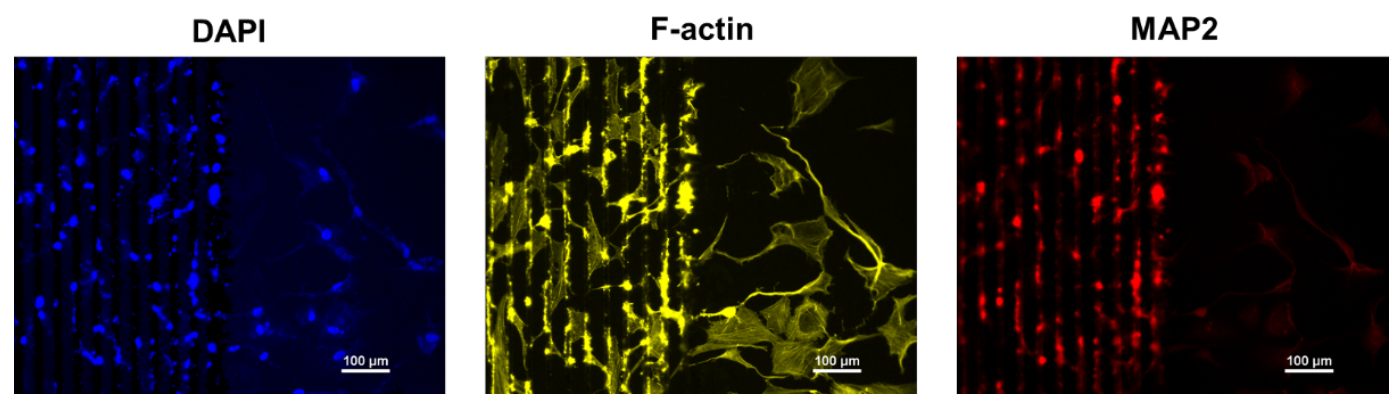

Figure S7. Representative fluorescence images of the HMSCs seeded on NM-RGD and flat surface in the same device. Only the HMSCs seeded on NME-RGD substrate show strong MAP2 signal. This control experiment proves the specificity of the MAP2 antibody. In addition, compared the HMSCs on flat surface, the HMSCs on NM-RGD substrate have significantly lower cell size.

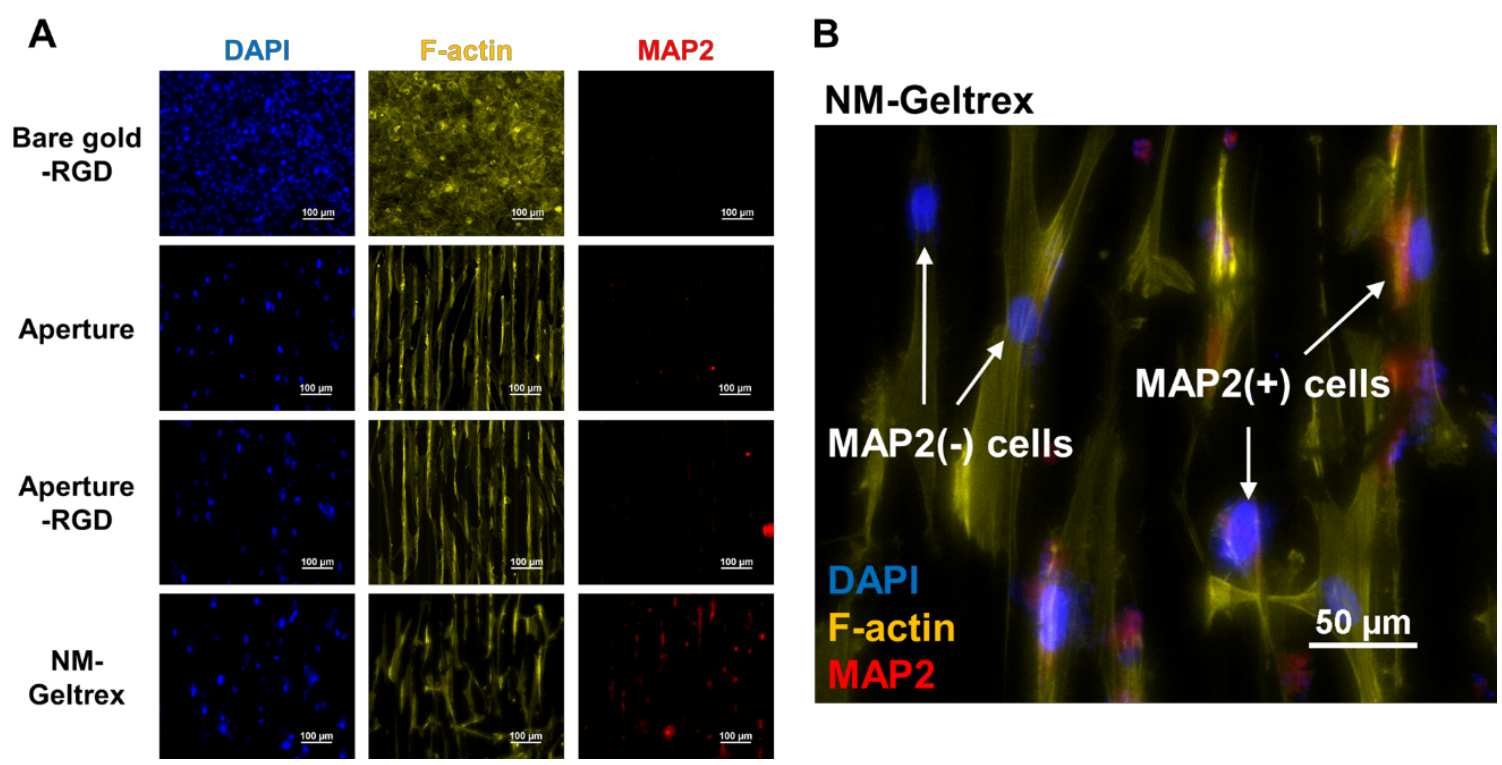

Figure S8. Effects of RGD or non-specific coating on neural differentiation of HMSCs. (A) Representative fluorescence images of the HMSCs seeded on various substrates for 7 days. (B) High-magnification images of the HMSCs seeded on the NMGeltrex substrate. 


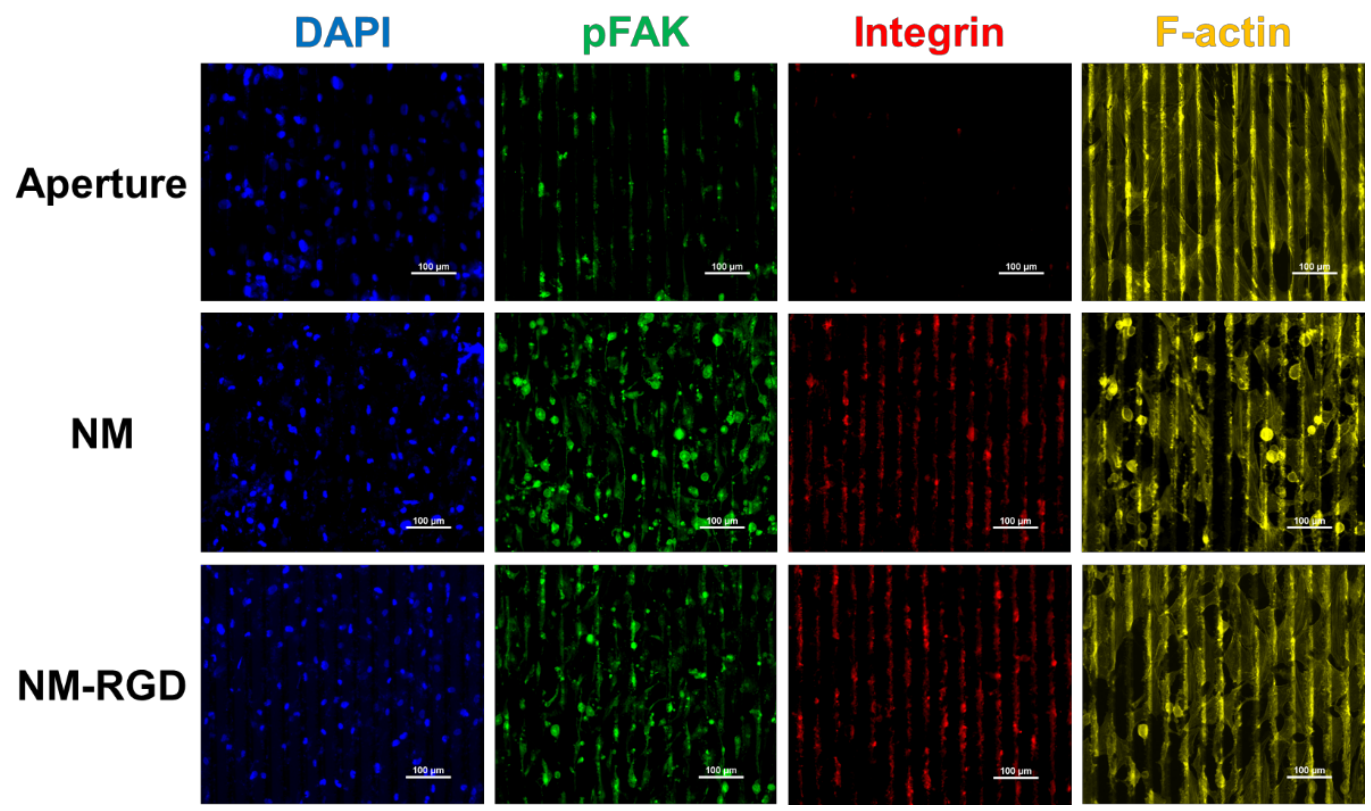

Figure S9. Representative fluorescence images of on-chip day10 HMSCs stained by pFAK, Integrin $\beta 1$ and F-actin. With the presence of nanostructured architectures, the HMSCs show significant higher expression of pFAK and Integrin.

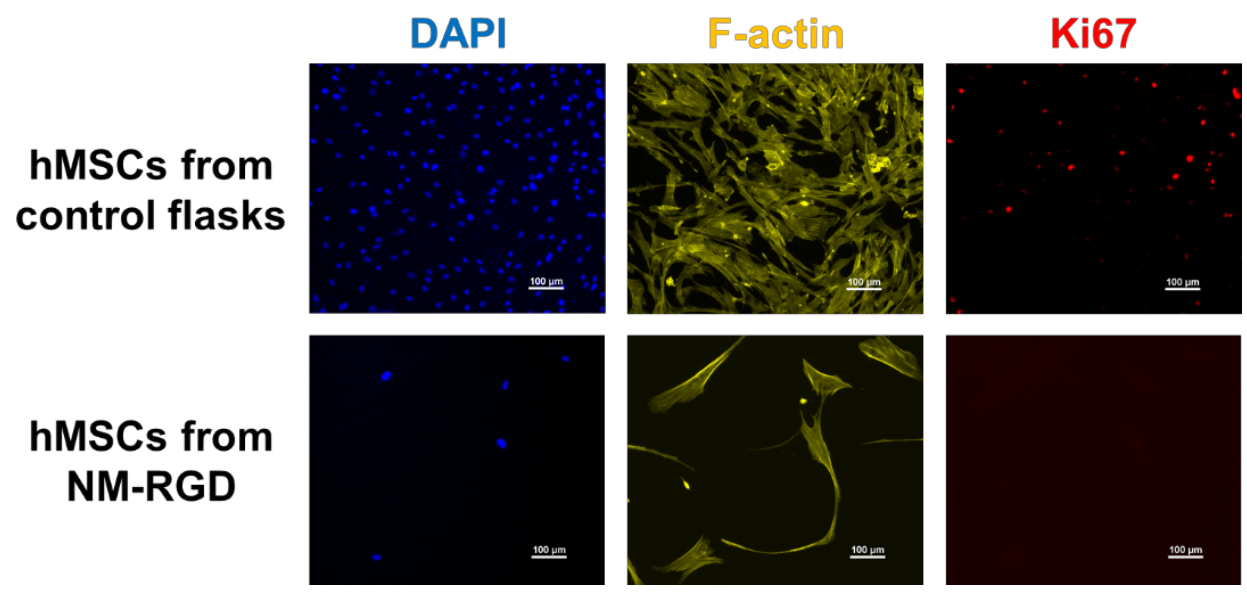

Figure S10. Representative fluorescence images of F-actin/Ki67 stained HMSCs ten days after release. Recovered HMSCs still elongated morphology and little proliferation, indicating they underwent permanent differentiation. 


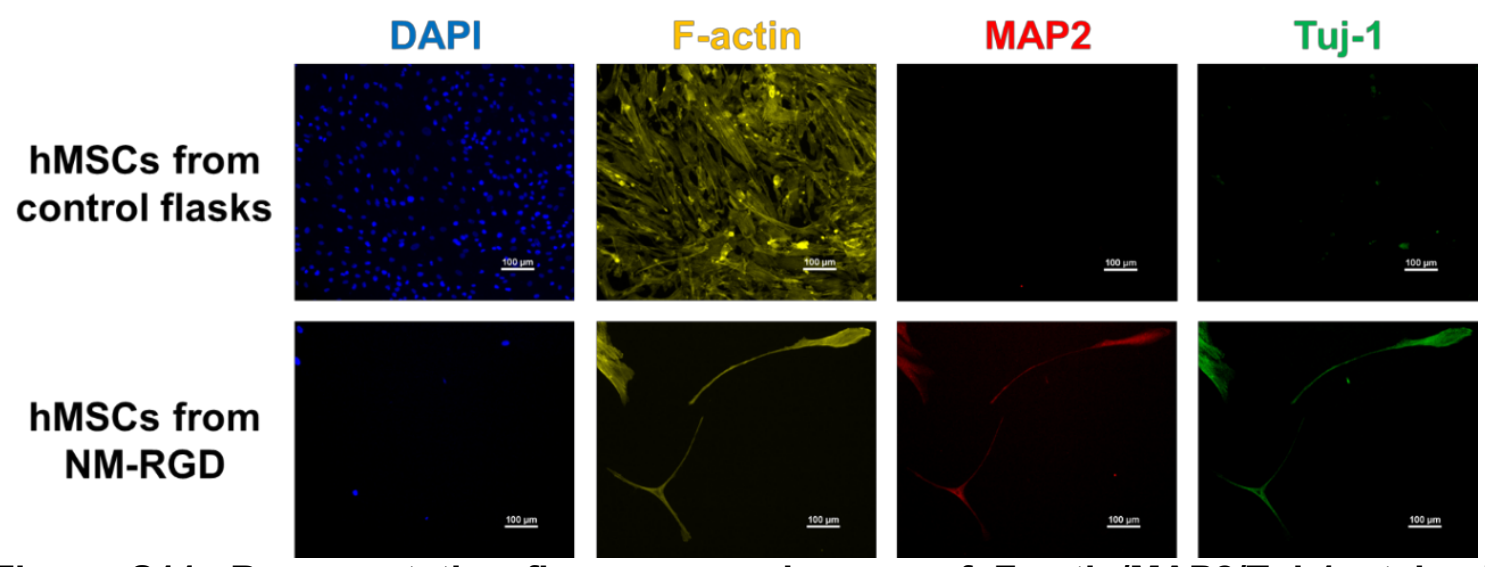

Figure S11. Representative fluorescence images of F-actin/MAP2/Tuj-1 stained HMSCs ten days after release. Released HMSCs still show strong expression of MAP2 and Tuj-1, indicating they underwent permanent differentiation. 